\title{
Presenting and visualizing image results for professional image searchers: A field evaluation
}

\author{
Ayşe Göker ${ }^{1}$, Richard Butterworth ${ }^{2}$, Andy MacFarlane ${ }^{2}$ and Simone Stumpf ${ }^{2}$ \\ ${ }^{1}$ Ambisense Ltd \\ ${ }^{2}$ Centre for $\mathrm{HCl}$ Design, School of Mathematics, Computer Science and Engineering, University of London
}

\begin{abstract}
Creative professionals such as journalists and copywriters are an important group of users who need to search for images as part of their work task. We describe the design and evaluation of a 'high density' image search interface targeted at this user group. We use a field evaluation together with a qualitative approach with creative professionals to gather feedback on the high density interface design. Our results show that creative professional viewed the interface favourably because they were able to get a quick overview of research results. We make suggestions for the appropriateness of high density interfaces for different work contexts, and further work in terms of layouts of images in high density interfaces.
\end{abstract}

Image retrieval, user interface, results presentation, professional image searchers, high density UIs

\section{INTRODUCTION}

Creative professionals, such as journalists, copywriters, marketers, web designers etc., face a nontrivial challenge in finding an appropriate image from the enormous number available within very tight deadlines. Typical use cases include illustrating text in fashion, graphic design, publishing and advertising (Eakins and Graham, 1999). There are a number of commercial image search engines, e.g. Getty Images. These systems provide a limited set of images to the user, often requiring the user to scroll through several screens of images with no overview of the result set arising from the search. We have evidence from prior work (Göker et al, 2016.) that some types of professional users find this limitation frustrating. Therefore, we propose an alternative approach that shows the image searcher many images at once i.e. a 'high density' image retrieval interface. This provides the user with more control over the retrieved items, within the constraints of screen real estate and usability. We hypothesize that this high density interface will be positively received by professional image searchers.

The paper is organized as follows. In section 2 we review the related work in image needs and visualization on the interface. We describe and justify the

(C) Goker et al. Published by BCS Learning and Development.

Proceedings of British HCI 2017 - Digital Make-Believe.

Sunderland, UK. interface design in section 3. Our experimental design for the field evaluation is described in section 4 , and the results of the evaluation are analyzed using qualitative methods in section 5 . We discuss the implications of this work in section 6 and conclude in section 7 .

\section{RELATED WORK IN IMAGE SEARCH}

There is some human-centred image information need and task analysis work as exemplified by Rodden et al. (2001) and studies that examine the contextual aspects of image information needs have emerged e.g. Chung and Yoon (2011). Rodden et al's work (2001) sheds light on how users view, search, and categorise images but there is generally a lack of this kind of insight for image visualizations, and the resulting overall system effectiveness in relation to users' image information needs.

Göker et al (2016) have reported the results of an interview study with several stakeholders in the photo library business, including image users, photographers and image curators in order to understand the different work tasks that stakeholders undertake with respect to images. Most of the participants described an initial "scoping out search" search by association which is based on Cox et al's (2000) categorization - until they formed a fair- 
ly specific target image in their heads and then working through many results from a search engine looking for an image that adequately matches the imagined target image. One of the most interesting findings was that interviewees repeatedly expressed irritation at having to trawl through a significant number of pages of results from image search engines or databases to get to the image they felt appropriate. This motivates our use of a high density interface showing many images in this paper.

There are many ways of laying out or presenting images on a database, and a survey of the methods is provided by Plant and Schaefer (2011), which includes basing the layout on features such as colour, visualization techniques such as clustering etc, and browsing by panning, zooming etc. Within these results images can be ordered by time, relevance, clusters hierarchies or graphs (Data et al, 2008). We use this prior work to inform the design of our interface in section 3 below.

\section{DESIGNING THE HIGH DENSITY IMAGE INTERFACES}

We displayed a large number of image search results as very small thumbnails, with two layouts: sunflower and honeycomb (see Figures 1 and 2). These designs were informed by a previous experiment which we used as a usability test for this study (Göker et al, 2017). We justify and explain these designs in this section.

A wide variety of different methods have been suggested for visualizing images (Yoshizawa, 2004; Hearst, 2009; Torres et al, 2003) including circles and other suggested by biological evolution such as the honeycomb. One particular method of displaying images on the screen is the sunflower pattern (figure 1), based on the ideas of Vogel (1979) and Rose (1999). This visual metaphor is focused on efficiently packing images on the screen, but is not necessarily pleasing to look at (figure 1) therefore a honeycomb pattern was also developed for contrast (figure 2).

For both layout types, relevant images were placed at the centre of the screen, with less relevant images displayed in concentric circles to the outside of the display. Relevance was determined by the IR system used for experiments, although in principle any method for relevance could be considered e.g. order by date. Users could 'zoom' in and out on the interface using a slider which allowed the users to expand the images from 30 pixels up to 150 pixels. Because the interface could now be zoomed this meant that as the zoom level increased more and more images would fall outside the zoom window, so the user was also allowed to drag the images around inside the zoom window. A small delay $(0.3$ seconds) on the expansion of the thumbnail when the mouse cursor hovered over the image.

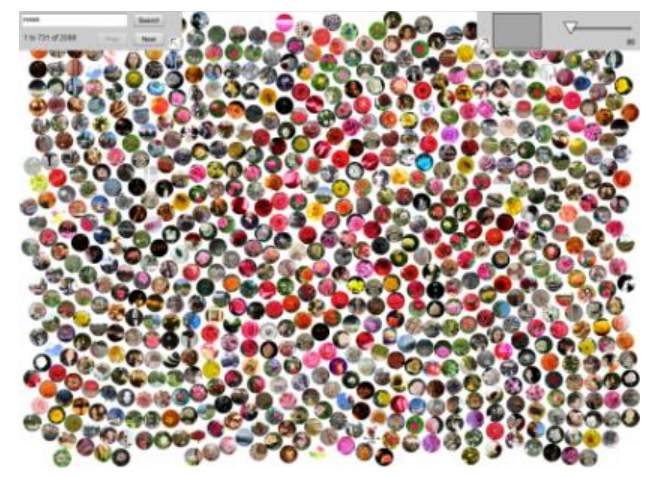

Figure 1. The sunflower arrangements with thumbnails at 30 pixels

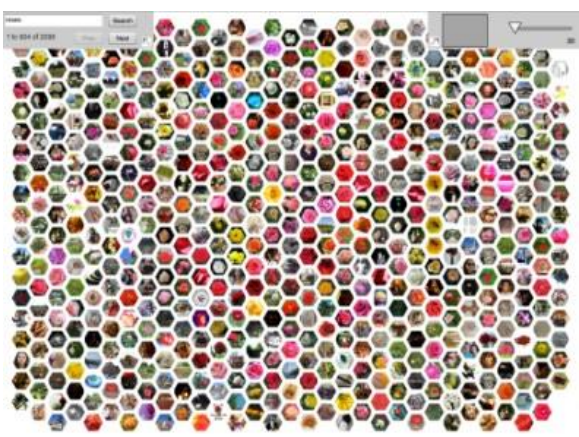

Figure 2. The honeycomb arrangements with thumbnails at 30 pixels

\section{EXPERIMENT DESIGN FOR THE FIELD STUDY}

In order to investigate whether high-density interfaces are suitable for creative professionals we, focused on the use of the high-density interface only in real search tasks based on real user needs.

Both interfaces were evaluated by semi-structured interviews with 10 participants and who have experience in jobs where image searching plays a predominant role. Four of the interviewees worked in marketing, two were website developers, three were advertising copywriters and one was a journalist.

Participants were asked to describe the way in which they searched for images as part of their normal work tasks, and to describe the image libraries they typically used. The interviewer then demonstrated the two high density image interfaces, describing the source of the images in the da- 
tabase and the way that the prototype related to its envisaged gesture based tablet interface. The interviewees were then invited to perform a typical image searching task using both of the interfaces and to articulate their thoughts about the interfaces as they did so. After the interviewees had explored the interface they were asked a set of follow-up questions relating to the usability of the interfaces and what they felt was good/bad or missing. Following the semi-structured nature of the interview, if the interviewees said something valuable or interesting, the interviewer would ask further questions on-the-fly to elicit more information. The interviewees were also explicitly asked to use the zoom function on the interface to set the thumbnails at the smallest useful size.

The interviewers made an explicit effort to encourage the interviewees to express their opinions, whether positive or negative. All the interviews were video recorded and took between twenty minutes and half an hour with one of ten minutes.

The raw data collected from the interviews was analysed using contextual inquiry, a modified Grounded Theory approach (Strauss and Corbin, 1998). This is an inductive approach which identified specific experiences of our participants, to reveal more general findings that seek to 'explain' how users find images to complete their work tasks. Transcripts from the interviews were analysed and categories identified e.g. filtering, interface design etc.

We used the Xapian system for experiments (Xapian, N.D.) which used the BM25 ranking function to determine relevancy (Robertson et, al, 1994) which determined the placement of images in the layout. The image collection used for this experiment was a subset of the CoPhIR image test set (Bolettieri et al, 2009). These crawled images from Flickr had textual descriptions in title, tags and comments fields which were indexed using the Xapian information retrieval system

\section{FIELD EVALUATION RESULTS}

All the interviewees broadly approved of the high density interface. None of the interviewees expressed an opinion that they would prefer using a traditional linear image retrieval interface over the prototype interface. Each interviewee was asked whether, given a choice between searching for images using a traditional interface, and the prototype, all other things being equal, which would they choose. None of the interviewees expressed an opinion that they would prefer a traditional inter- face. The responses ranged from neutral (four interviewees) to a definite preference for the high density interface (six interviewees). In the case of the neutral responses the interviewer probed as to what would be the deciding factor between two different image retrieval interfaces, but did not receive clear cut answers, and it is likely that the participants did not wish to be pinned down to such a clear cut decision. Furthermore all the interviewees agreed that the potential benefits of putting a lot of images on screen outweighed the drawbacks. Of the interviewees who responded most positively to the prototype high density interface, most expressed a clear opinion that a high density interface would cut out the need to trawl through page after page of results. This was a particularly satisfying result, as those interviewees were seeing (without prompting from the interviewer) that the system did in fact solve the problem that we were initially motivated to solve. This overall positive response to the prototype interface came with many caveats however, relating to the interface design, interaction with image sets and display of individual images with useful suggestions provided by the participants. We examine these issues below.

In terms of the interface design the honeycomb arrangement was preferred over the sunflower arrangement, with all interviewees expressing preference for the honeycomb. The regularity of the honeycomb pattern meant that the users could systematically look at each image. The layout of the sunflower was felt to be to at best 'too random' and at worst to be painful to look at (particularly when the thumbnails were very small). In terms of placing the most relevant images within the layout, all interviewees either preferred or were neutral about the placing of the most relevant images centrally with the less relevant images radiating outwards. However none of the interviewees realised that this was the case until it was explained to them, and although they agreed that this was an acceptable or sensible way of organising the images, they would prefer if that organization was somehow signaled to them, for example by making the most relevant images slightly larger, or by including some sort of background concentric 'heat map' where the most relevant images are shown on a red background, and the less relevant the images become, the colder the background colour becomes. Further refinements could include clustering similar images - suggested by several interviewees. This is consistent with Rodden's (2002) findings. There was no particular consensus on appropriate ways of clustering; colour was suggested, so that a predominant colour was extracted from the images and then the images could be ar- 
ranged on the screen in a colour wheel. A more sophisticated suggestion was to cluster images according to similarity in tags, and then allow the user to select a particular image and then perform a new 'query by example' search on that image, so that user can incrementally gather a collection of images that are (hopefully) nearer and nearer to the target image. The issue of filtering was mentioned by the participant (functionality which can be quickly added to the UI), and contradictory opinions were expressed on zooming - some users were positive some negative on this functionality.

Participants raised an issue with the display of individual images in terms what and how much of the images should be displayed. Several interviewees expressed concern with the amount of cropping that the high density interface did to the image. They stated that they were not able to see if the image was in landscape or portrait orientation without mousing over the image, and that orientation was an important factor in their image requirements. Even when we then discussed the possibility of filtering for orientation, several of the interviewees still expressed a wish to be able to see more of the image. All the interviewees expressed a preference for a smallest thumbnail size of between 50 and 70 pixels, with the majority choosing around 60 pixels. None of the interviewees saw value in having thumbnails smaller than these sizes. The interviewer would then show the search results at 30 pixels and suggest that this might be useful to give an overview of what was available in the database, but most interviewees disagreed with this, or were unsure.

In conclusion the interviewees agreed that the prototype interface would be valuable in their day to day tasks, and most could not think of any tasks where the prototype interface would be particularly inappropriate, though one interviewee did state that the high density interface would not be appropriate for showing image selections to clients.

\section{DISCUSSION}

It was apparent that several of the interviewees felt that the high density interface had a 'wow' factor, particularly caused by the initial animation which zoomed the search results from 30 to 60 pixel thumbnails. Although this initial animation was included to give users a feel for the size of the result set while not deluging then in several hundred images, it did have the effect of giving the interface a very dynamic feel, rather than the static linear lists of images generated by traditional image retrieval interfaces. It could therefore be the case that the initial impression given by the interface caused the interviewees to have an inflated opinion of the capabilities of the interface.

As it stands the interface still requires some work but we believe that we have validated the concept of a high density image retrieval interface sufficiently such that a further test on the prototype design would be fruitful. From a research point of view we feel that we have sufficient evidence to support our hypothesis that the benefits of a high density interface for professional image searchers outweigh the disadvantages. Our research has also shown that the high density interface needs to be carefully designed; an interface where lots of images are simultaneously displayed is not necessarily going to be a success. The images need to be spaced out, organised into a regular pattern and although they can be cropped to improve the way that they are packed on the screen, this cropping needs to be done more carefully. In tasks and applications which present the user with the problem of sifting through large numbers of images, we have evidence that a high density interface is at least an acceptable and useful mitigation of the problem of sorting through many images. Overall it was apparent during the interviews that the interviewees 'got' the idea of the high density interface, with several expressing positive opinions about the reduced need for trawling through many pages of results.

\section{SUMMARY AND CONCLUSION}

In this research we have used a field evaluation approach to investigate the design and evaluation of an image search interface for professional users. We used this method as the cohort was hard to access who are time poor and would not take time out to undertake a formal experiment without being remunerated at professional rates - beyond the budget of our project.

In terms of the design itself, our original purpose was to address the needs of professional users who require access to high volumes of images, suggested by prior work (Göker et al, 2016). The hypothesis here was that a high density interface would serve the needs of these professional users, who have built up significant tacit knowledge in sifting images through their daily work tasks. The evidence produced here is that there is evidence that such an interface would be accepted by that user group and our main contribution here is the final design produced.

It was clear from the evaluation that high density interfaces are not universally applicable to all im- 
age retrieval scenarios - professional users would not use high density interfaces to display images to clients. Clearly the work context and task is an important consideration when thinking about an appropriate design for image retrieval users. This is a further refinement to our main contribution - the design is only applicable to users who have the cognitive abilities to process high volumes of images. A further refinement was the issue of density of images versus aesthetics, by using sunflower and honeycomb metaphors for image layouts. From the evidence we have here it is clear that users preferred the honeycomb layout - therefore the evidence we have (albeit limited) is that effective image seeking using a high density image interface is driven more by aesthetics. However there is a lot more work which needs to be done in this area, in particular the investigation of the many different layouts identified in the literature (Yoshizawa, 2004; Hearst, 2009; Torres; 2003) and to provide more concrete evidence of the preference for density as against aesthetics.

\section{ACKNOWLEDGEMENTS}

This work is supported by the Piclet project, funded by the TSB reference TP14/DIC/6/I/BL209H.

\section{REFERENCES}

Bolettieri, P., Esuli, A., Falchi, F., Lucchese, C., Perego, R., Piccioli, T., Rabitti, F. (2009) CoPhIR: a Test Collection for Content-Based Image Retrieval. CoRR abs/0905.4627.

Chung, E.K., and Yoon, J.W. (2011) Image needs in the context of image use: An exploratory study. J. Info. Sci. 37, 163-177.

Cox, I. J., Miller, M. L., Minka, T. P., Papathomas, T. V., and Yianilos, P. N. (2000) The Bayesian Image Retrieval System, PicHunter: Theory, Implementation, and Psychophysical Experiments. IEEE Transactions on Image Processing. 9, 2037.

Datta, R., Joshi, D., Li, J., and Wang, J.Z. (2008) Image Retrieval: Ideas, Influences, and Trends of the New Age. ACM Comp. Sur. 40, 5:1-60.

Eakins, J.P., and Graham, M. (1999) Contentbased image retrieval. JISC Technology applications program report: 39. https://tinyurl.com/hxkhbxm (Last accessed: $9^{\text {th }}$ February 2017).
Göker, A., Butterworth, R., MacFarlane, A., Ahmed, T and Stumpf, S. (2016) Expeditions through image jungles: a study of the commercial use of image libraries. J. Doc, 71, 5-23.

Göker, A., Butterworth, R., MacFarlane, A., Ahmed, T and Stumpf, S. (2017). Presenting and visualizing results on an image retrieval user interface. Proceedings of British $\mathrm{HCl}$ (in this volume).

Hearst, M. (2009) Search User Interfaces. Chapter 10: Information visualization for search interfaces. Cambridge University Press.

Plant, W., and Schaefer, G. (2011) Visualisation and browsing of Image Databases. In Multimedia Analysis, Processing and Communications, Studies in Computational Intelligence 346, Lin, W. Tao, D., Kacprzyk, J., Izquierdo, E. and Wang, H. (eds). Springer-Verlag, pp 3-57.

Rodden, K., Basalaj, W., Sinclair, D., and Wood, K. (2001). Does organisation by similarity assist image browsing? Proc. CHI 2001. pp. 190-197, ACM Press, New York.

Rodden, K. (2002) Evaluating similarity-based visualisations as interfaces for image browsing. University of Cambridge, Computer Laboratory. Technical Report No 543. UCAM-CL-TR-543.

Robertson, S., Walker, S., Jones, S. HancockBeaulieu, M., and Gatford, M. (1994) Okapi at TREC-3. Proc. TREC-3. pp 109-126. NIST Gaithersburg.

Rose, S.J. (1999) The Sunflower visual metaphor, A new paradigm for dimensional compression. In: IEEE Symposium on Information Visualisation, 128-131, IEEE Press, Hoboken, N.J.

Strauss, A. L., \& Corbin, J. M. (1998) Basics of Qualitative Research: Techniques and Procedures for developing Grounded Theory. Sage publications.

Torres, R.S., Silva, C.G., Medeiros, C.B. and Rocha, H.V. (2003) Visual Structures for Image Browsing, In: Proc. CIKM 2004, pp 49-55. ACM Press, New York.

Vogel, H (1979) A better way to construct the sunflower head. Math. Biosci. 44, 179-189.

Xapian Project. https://xapian.org/ (Last accessed: $9^{\text {th }}$ May 2017).

Yoshizawa, T. (2004) User interface techniques for browsing and searching image databases. Doctoral dissertation. University of Texas at Dallas, USA. 